\title{
A phase Ib dose allocation study of oral administration of lucitanib given in combination with fulvestrant in patients with estrogen receptor-positive and FGFR1-amplified or non-amplified metastatic breast cancer
}

\author{
M. Campone ${ }^{1} \cdot$ T. Bachelot ${ }^{2} \cdot$ F. Penault-Llorca ${ }^{3} \cdot$ A. Pallis ${ }^{4} \cdot$ V. Agrapart ${ }^{4} \cdot$ M. J. Pierrat ${ }^{4} \cdot$ C. Poirot $^{4} \cdot$ F. Dubois ${ }^{4}$.

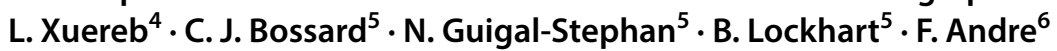

Received: 29 November 2018 / Accepted: 27 December 2018 / Published online: 25 January 2019

(c) The Author(s) 2019

\begin{abstract}
Purpose The primary objective of this multicentric dose allocation and dose expansion study was to determine the MTD and the DLTs of the lucitanib (a tyrosine kinase inhibitor of the FGFR/VEGFR/PDFGR pathways)/fulvestrant combination. Methods Postmenopausal women with ER+/HER2- mBC, who have relapsed during or after treatment with fulvestrant, were eligible. The study had a dose allocation part to assess the tolerability of the combination followed by a dose expansion part.

Results Eighteen patients with ER+, mBC were enrolled; median age was 66 years, $50 \%$ had a PS: 0 and all had received previous endocrine treatment. The study was prematurely terminated after 18 patients (15 in part 1 and 3 in part 2) based on preclinical experiments that failed to confirm the hypothesis that addition of lucitanib would reverse sensitivity to endocrine treatments. Based on data of global lucitanib development, it was decided to stop the dose allocation at $12.5 \mathrm{mg}$ and to start the dose expansion part at $10 \mathrm{mg} /$ day. The most common grade $\geq 3$ toxicities ( $>10 \%$ of patients) were hypertension (78\%) and asthenia (22\%). All patients required at $\geq 1$ interruption, 13 patients $(72 \%)$ required $\geq 1$ dose reduction. Three patients (72\%) withdrew from the study for AEs (at $10 \mathrm{mg}$ ). Three patients achieved a confirmed PR (10 $\mathrm{mg} n=1 ; 12.5 \mathrm{mg} n=2)$. Conclusion Although the combination is feasible it requires close monitoring of the patients for the management of adverse events. Further investigation is required to better understand the potential role of FGFR inhibition in reversing resistance to endocrine treatment.
\end{abstract}

Keywords Metastatic breast cancer $\cdot$ Hormone receptor positive $\cdot$ FGF pathway $\cdot$ VEGF pathway lucitanib $\cdot$ Fulvestrant

Electronic supplementary material The online version of this article (https://doi.org/10.1007/s00280-018-03765-3) contains supplementary material, which is available to authorized users.

M. Campone

mario.campone@ico.unicancer.fr

1 Institut de Cancérologie de l'Ouest-Centre René Gauducheau, Saint-Herblain, France

2 Centre Léon Bérard Centre de Lutte Contre le Cancer (CLCC) de Lyon, Lyon, France

3 Centre Jean Perrin, Clermont-Ferrand, France

4 Institut de Recherches Internationales Servier, Suresnes, France

5 Institut de Recherches Servier, Croissy-sur-Seine, France

6 Institut Gustave Roussy, Villejuif, France

\section{Introduction}

The fibroblast growth factor receptor (FGFR) family consists of four, membrane-bound, tyrosine kinase receptors (FGFR 1-4) [1]. The FGFR receptors are activated by binding of 22 structurally similar ligands [1]. Upon ligand binding to the extracellular domain of the receptors, the receptor undergoes dimerization, which enables transphosphorylation of a tyrosine in the activation loop of the kinase domain [2] leading to subsequent activation of several downstream molecules such as phosphoinositide-3 kinase (PI3K), extracellular signal-regulated kinase $1 / 2$ (ERK1/2), various signal transducer and activator of transcription (STAT) proteins, and protein kinase $\mathrm{C}$ (PKC) [1]. FGFR signaling produces distinct biological responses in different cell types, ranging 
from stimulation of cell proliferation and survival to growth arrest, migration, and differentiation [3].

Several different aberrations of the FGFR pathway have been described in breast cancer. Amplification FGFRI gene is observed in approximately $7.5-17 \%$ of all breast cancer and in 16-27\% of luminal B-type [4-10]. FGFRI amplification is considered a negative prognostic factor for metastasis free and overall survival in patients with estrogen receptor (ER) positive breast cancer (BC) [10-13]. Similarly, amplification of FGFR2 has been identified in approximately $4 \%$ of triple-negative breast cancers (TNBCs) [14]. Amplification of FGF ligands located on chromosome 11q13, including FGF3, FGF4 and FGF19, has also been observed in $15 \%$ of human breast cancers [15]. It is still not quite clear whether these ligands have the capacity to act as tumor drivers or if additional oncogenic changes are required. However, what is clear is that all of these ligands activate FGFR1 and/or FGFR2, which are both involved in breast cancer tumorigenesis and progression [1].

Several preclinical studies have suggested that targeting FGFR1 in FGFR1-amplified cell lines leads to anti-tumor effects. Shi et al. [16] have shown that dovitinib, an FGFR1-3 inhibitor, inhibits proliferation of $F G F R I$-amplified breast cancer cell lines, but not the FGFR non-amplified ones. Similar results were reported in another study showing that brivanib, another FGFR1 inhibitor, inhibits FGF-induced cell proliferation in FGFRI-amplified cell lines [17].

Moreover, alterations of the FGFR pathway have been implicated in mechanisms of resistance to endocrine treatment in breast cancer. Turner et al. have shown that FGFR1 overexpressing and amplified $\mathrm{BC}$ cell lines demonstrate enhanced ligand-dependent signaling, increased activation of MAPK and PI3K-AKT signaling pathways in response to FGF2. These cell lines also display basal ligand-independent signaling, and depend on FGFR signaling for anchorageindependent growth. FGFRI-amplified cell lines are resistant to 4-hydroxytamoxifen, while silencing of FGFR1 by small interfering RNA leads to reversal of resistance, an observation implying that FGFR1 overexpression is involved in endocrine therapy resistance [18]. Similarly, a case report by Balko et al. [19] also suggested that resistance to letrozole could be linked to FGFRI amplification in breast tumor. Recently, a study by Formisano et al. [20] has demonstrated that in a cohort of patients with $\mathrm{ER}+\mathrm{BC}$ that were treated with letrozole, FGFRI amplified tumors still sustained their proliferation despite pharmacological deprivation of estrogens. FGFR1 tied with ER $\alpha$ to drive estrogen-independent transcription of ER $\alpha$-responsive genes. The coupling of FGFR1 with ER $\alpha$ was subdued upon transfection with a FGFR 1 mutant that lacked kinase activity and by pharmacological inhibition of FGFR1. Finally, double blockage of FGFR1 and ER $\alpha$ with fulvestrant and FGFR tyrosine kinase inhibitors blocked the association of FGFR1 with ER $\alpha$ and suppressed growth of ER+/FGFRI - amplified PDXs [20].

Lucitanib is a potent, selective inhibitor of the tyrosine kinase activity of vascular endothelial growth factor receptors, types 1, 2, and 3 (VEGFR1-3); fibroblast growth factor receptors, types 1, 2, and 3 (FGFR1-3); and platelet-derived growth factor receptors, types alpha and beta $(\operatorname{PDGFR} \alpha / \beta)$ [21]. Lucitanib was evaluated in an open-label phase I/IIa first-in-human study that comprised of a dose-escalation phase (to determine maximum tolerated dose (MTD), recommended dose (RD), and pharmacokinetics of lucitanib in patients with advanced solid tumors), followed by a dose-expansion phase to get preliminary signs of efficacy in patients who could potentially experience clinical benefit from treatment [22]. Clinical activity was observed at all doses tested and in assessable FGF-aberrant BC patients overall response rate was $50 \%$ (6 out of 12 patients) while median PFS was 40.4 weeks for all treated patients [22].

These data demonstrating the role of FGFR pathway in the development of endocrine resistance as well as the encouraging clinical data observed with lucitanib in patients with $\mathrm{ER}+\mathrm{BC}$, form the rationale to evaluate the combination of lucitanib with fulvestrant in patients with $\mathrm{BC}$ resistant to endocrine therapy. The lucitanib/fulvestrant combination could potentially overcome the endocrine resistance in this patient population and delay the need for chemotherapy administration which is associated with more severe toxicity. The objective of this manuscript is to present the results of a phase Ib dose allocation study of oral administration of lucitanib given in combination with fulvestrant in patients with estrogen receptor-positive and FGFRI-amplified or non-amplified metastatic breast cancer.

\section{Patients and methods}

\section{Study design}

This was a multicentric, open, non-comparative, dose allocation and dose expansion study. A modified Continual Reassessment Method (mCRM) was used for the first part of this study (dose allocation). The target toxicity rate of 16-33\% (that is the dose for which 16-33\% of patients experienced a DLT) has been chosen between the sponsor and the investigators. For dose allocation cohorts, a minimum of three patients who met the eligibility criteria were to be enrolled at the initial dose level of $10 \mathrm{mg}$ once per day in combination with fulvestrant. Patients were to be included by groups of three or six, based on the decision taken at the previous end of cohort meeting. A minimum of nine patients were to be included at the MTD. Before testing a new dose level, a meeting between the Sponsor, the coordinator, the investigators, safety experts (a nephrologist, a cardiologist, an 
hepatologist) and an independent statistician took place to discuss the toxicities in terms of DLT, safety and PK data observed in patients, and to decide jointly the next dose level to be tested.

The dose allocation part was to be followed by a dose expansion part. In this second part 2, cohorts were to be opened: cohort A with $14 F G F$ amplification positive MBC patients (receptor or ligand amplified), and cohort B with 14 FGF non-amplified MBC patients.

Study design is presented in Fig. 1.

\section{Patients}

Eligible patients for this study were menopausal women, $\geq 18$ years old with an estimated life expectancy $>12$ weeks with histologically confirmed, stage IV, ER positive, HER2 negative breast adenocarcinoma that have progressed during or after treatment with fulvestrant. Patients also have to had ECOG performance status $<2$ and adequate haematological, hepatic and renal functions. The presence of an accessible metastatic lesion for biopsy was required for participation (expansion cohorts only). Patients with more than three chemotherapy regimens in the metastatic/advanced setting were not eligible for this study, as were patients with active central nervous system metastases, as indicated by clinical symptoms, cerebral oedema, and/or progressive growth.

In expansion cohorts, FGFR1 and $11 q 13$ testing was assessed at a central facility on a newly obtained or available archived metastatic biopsy during the patient inclusion period. FGFRI and $11 \mathrm{q} 13$ amplicon amplification status was assessed by FISH using probes of ZytoVision specific for the genomic regions of the FGFRl gene at 8p11 and CCND1, FGF3, FGF4, FGF19 at 11q13. FGFR1 amplification was defined as:

- FGFR1/CEN8 ratio is $\geq 2.0$; or

- Average number of $F G F R 1$ signals per tumor cell nucleus is $\geq 6$

$11 q 13$ amplicon amplification was defined when at least one of the following criteria is met:

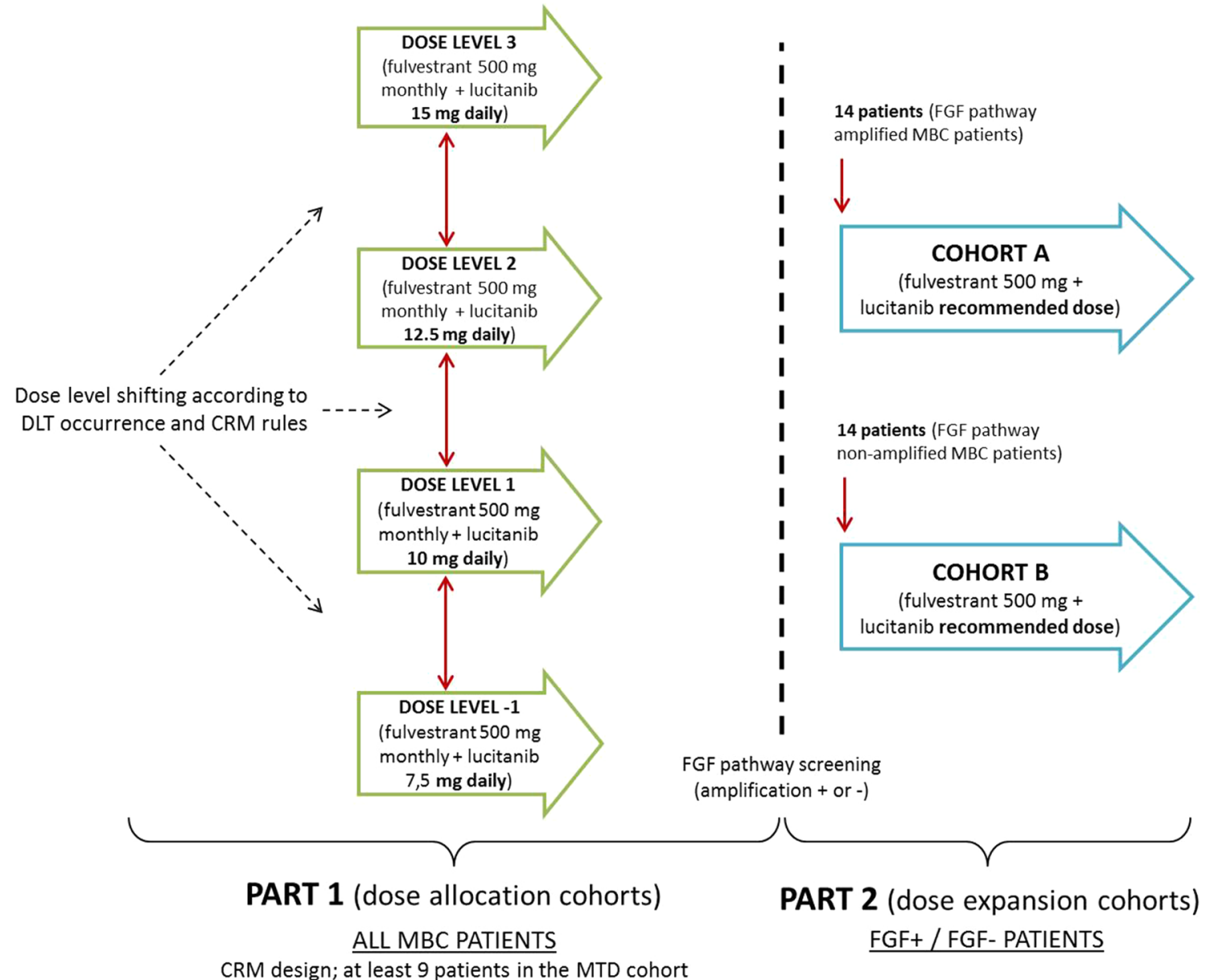

Fig. 1 Study design 
- $C C N D 1 / C E N 11$ ratio is $\geq 2.0$; or

- Average number of $C C N D 1$ signals per tumor cell nucleus is $\geq 6$

All patients gave written informed consent to participate in the study, and the trial was approved by the Ethics and Scientific Committees of the participating centers. The study was conducted according to the Helsinki Declaration and Good Clinical Practice guidelines [23].

\section{Treatment}

Eligible patients in both parts of the study patients received fulvestrant $500 \mathrm{mg}$ intra-muscular once per cycle (28 days) and lucitanib at their cohort starting dose $(7.5 \mathrm{mg}, 10 \mathrm{mg}$, $12.5 \mathrm{mg}$ or $15 \mathrm{mg}$ ) orally on a daily basis until unacceptable toxicity, disease progression or withdrawal of consent. Daily dose could be reduced down to $5 \mathrm{mg}$ in case of toxicity.

First, fulvestrant dose was administered at C1D1 and then every 28 days (every cycle), while the first lucitanib dose was administered on day 15 of cycle 1 . For patients who were not treated with fulvestrant for more than 2 months prior inclusion, an additional injection of fulvestrant $500 \mathrm{mg}$ was administered at C1D15.

\section{Objectives}

The primary objectives of this study were to determine the maximum tolerated dose (MTD) and the dose limiting toxicities (DLTs) of the lucitanib/fulvestrant combination and to identify the recommended Phase II dose.

Secondary objectives included the measurement of tumor response (RECIST v1.1 [24]) and the determination of the pharmacodynamic (PD) profile of lucitanib.

\section{Assessments}

DLTs occurring between C1D15 and C2D15 (first 28 days of administration of the combination lucitanib + fulvestrant) were assessed on C2D15 (in both parts of the study). DLT definition included grade 3 febrile neutropenia or prolonged grade 4 neutropenia (ANC $<0.5 \times 10^{9} / \mathrm{L}$ for more than 5 days), grade 3 thrombocytopenia lasting more than 5 days or any other grade 4 haematological toxicities. All grade $\geq 3$ non-haematologic toxicity (grade 3 increase in AST and ALT lasting for more than 7 days; hypertension with $\mathrm{SBP} \geq 160 \mathrm{mmHg}$ or $\mathrm{DBP} \geq 100 \mathrm{mmHg}$ if not controlled by antihypertensive therapy within 7 days; grade 3 nausea and vomiting if not controlled by antiemetic treatment) and grade 2 proteinuria lasting more than 7 days were also considered as DLT. Finally, administration of less than $75 \%$ of planned doses of lucitanib due to treatment related toxicities was also considered as a DLT.

For evaluation of tumor response, all patients in both parts of the study had a baseline tumor evaluation (RECIST 1.1) performed within 28 days before the first administration of fulvestrant for the study. Radiological and clinical tumor measurement was then repeated every two cycles.

A metastatic tumor sample was obtained at baseline (optional in allocation part, mandatory in expansion part) to analyze predictive biomarkers for response to lucitanib. FGFR1 amplification and 11q13 amplicon amplification were evaluated centrally using ZytoVision commercial probes and scored (ratio on centromere, copy numbers per nucleus) by a team of independent pathologists. FGFR1 expression level was evaluated centrally by IHC (using a rabbit FGFR1 antibody from Abcam), and scored (H-score-total-membrane-cytoplasm) by an independent pathologist. A primary tumor sample was optionally collected to perform similar analysis.

Blood samples were also collected at C1D15 (prior lucitanib first intake) and at C2D1 (predose, on lucitanib treatment) for analysis of predictive biomarkers for lucitanib response or lucitanib biological activity: serum FGF2, FGF23, VEGFA and other markers of angiogenesis.

\section{Statistical analysis}

All analyses were performed with SAS (version 9.2; SAS Institute, Cary, NC) except for the mCRM analysis which was performed with $\mathrm{R}$ (version 2 or later). Descriptive statistics (continuous data) or contingency tables (categorical data) were used. The survival functions of the time dependent parameters were estimated via Kaplan-Meier curves. All analyses were provided by initial dose level and for all patients. The MTD was determined in patients who received the right dose assigned and completed the safety evaluation or discontinued treatment due to a DLT.

For pharmacodynamics analysis, the change from baseline to C2D1 (after 15 days of Lucitanib treatment) was test by a Wilcoxon paired test followed by a FDR correction (Benjamini-Hochberg) to correct for multiplicity.

\section{Results}

\section{Patient demographics}

From 10th of April 2014 to 6th of March 2017, a total of 18 patients with ER positive, metastatic BC were enrolled into the study. Patient characteristics are presented in Table 1. The median age at enrollment was 66 years, median duration of metastatic $\mathrm{BC}$ was 3.5 years and median time since last progression was 0.8 months. Half of the patients had a PS 
Table 1 Patients' characteristics

\begin{tabular}{llll}
\hline & $10 \mathrm{mg}(N=12)$ & $12.5 \mathrm{mg}(N=6)$ & All $(N=18)$ \\
\hline Age (years) & & & \\
Median & 64.00 & 66.50 & 66.00 \\
Min-max & $46.0-78.0$ & $52.0-76.0$ & $46.0-78.0$ \\
$>65[n(\%)]$ & $6(50.0)$ & $4(66.7)$ & $10(55.6)$ \\
Performance status & & & \\
Nobs & 11 & 5 & 16 \\
0 & $6(54.6)$ & $2(40.0)$ & $8(50.0)$ \\
1 & $5(45.4)$ & $3(60.0)$ & $8(50.0)$ \\
Time since diagnosis of metastatic disease (years) & & & 3.5 \\
Median & 3.4 & 4.4 & $0.8-8.8$ \\
Min-max & $0.8-7.7$ & $1.5-8.8$ & \\
Time since latest progression (months) & & & 0.8 \\
Median & 0.8 & 0.9 & $0.3-3.9$ \\
Min-max & $0.3-3.9$ & $0.5-1.4$ & $18(100)$ \\
Previous endocrine treatment $[n(\%)]$ & $12(100)$ & $6(100)$ & $18(100)$ \\
Previous treatment with fulvestrant $[n(\%)]$ & $12(100)$ & $6(100)$ & $8(94)$ \\
Previous chemotherapy treatment $[n(\%)]$ & $11(92 \%)$ & $6(100)$ & $4(50)$ \\
FGFR1 amplification ${ }^{\mathrm{a}}$ & & & \\
Nobs & 7 & 1 & $1(100)$ \\
$n(\%)$ & $3(43)$ & & \\
\hline
\end{tabular}

${ }^{a}$ FGFR1 pathway amplification (FGFR1 and/or 11q13) on primary tissue and/or metastatic biopsy

of 0 . All patients had received previous endocrine treatment, while almost all (94\%) had received previous chemotherapy and approximately two-thirds had received three or more previous regimens of treatment for metastatic disease. All patients were evaluable for toxicity and response.

In September 2015, following a discussion between the principal investigator and the sponsor it was commonly decided to prematurely terminate this study after 18 patients (15 in part 1 and 3 in part 2) have been enrolled. This decision was based on preclinical experiments completed by the sponsor (Unpublished Data) that failed to confirm the hypothesis that addition of lucitanib would reverse sensitivity to endocrine treatments. Moreover, data from other lucitanib monotherapy studies in breast cancer demonstrated moderate activity compared to standard of care and failed to confirm a predictive role for FGFR amplification [EudraCT nos. 2013-000288-10 (FINESSE) and NCT02109016 (US Breast)].

\section{Dose limiting toxicities and recommended dose}

During the dose allocation part of the study (part 1), patients were included by cohort of 3 or 6 patients. In the first cohort of three patients treated at $10 \mathrm{mg} / \mathrm{day}$, one patient reported one DLT (grade 3 hypertension: hypertension with SBP $\geq 160 \mathrm{mmHg}$ or DBP $\geq 100 \mathrm{mmHg}$ not controlled to SBP $<160 \mathrm{mmHg}$ and DBP $<100 \mathrm{mmHg}$ by antihypertensive therapy within 7 days after optimization of antihypertensive therapy).

According to the mCRM design, one additional cohort of three patients (cohort no. 2) was included at the same dose level of $10 \mathrm{mg} / \mathrm{day}$, with no DLT reported in these three patients. In accordance with the mCRM design and taking into account clinical information from the 6 patients as well as accumulating safety data from 135 patients that were treated at that time in the whole lucitanib clinical development program, 1 additional cohort of 3 patients (cohort no. 3) treated at the $10 \mathrm{mg} /$ day dose level was included for whom no other DLT was reported.

Since the mCRM recommended the dose of $12.5 \mathrm{mg} /$ day and taking into account clinical information from the first nine patients included (cohorts nos. 1, 2 and 3), it was confirmed that the dose level of $10 \mathrm{mg} /$ day was well tolerated, and that the escalation of the next dose of $12.5 \mathrm{mg} /$ day was appropriate. Therefore, it was decided to include six patients at the dose level of $12.5 \mathrm{mg} /$ day (cohort no. 4). No new DLT was reported for those six patients.

Taking into account the DLT information from the nine patients treated at $10 \mathrm{mg} /$ day and the six patients treated at $12.5 \mathrm{mg} /$ day of lucitanib in combination with fulvestrant, the $\mathrm{mCRM}$ recommended the dose of $15 \mathrm{mg} /$ day for the next cohort. However, a pooled analysis, done on data from five lucitanib studies [EudraCT nos. 2013-00028810 (FINESSE), 2010-019121-34 (FIH), 2013-001520-19 (INES) and 2013-003874-29 (Lung) and NCT02109016 
(US Breast)], showed that the $10 \mathrm{mg} /$ day would be a better tolerated dose (emergent adverse events appeared to be less frequent at $10 \mathrm{mg}$, especially grade 3 hypertension). Based on these safety considerations, it was decided to stop the dose allocation (part 1 of the study) and to start the dose expansion part (part 2) at $10 \mathrm{mg} /$ day. The recommended dose of lucitanib (given in combination with fulvestrant) for part 2 of the study was determined at $10 \mathrm{mg} /$ day. No new DLT was observed within the three patients included in the expansion part.

\section{Treatment exposure}

Median duration of lucitanib and fulvestrant treatment was 28.1 weeks (24.6 weeks in the $10 \mathrm{mg}$ dose level and 44.6 weeks in the $12.5 \mathrm{mg}$ dose level), while the median treatment duration for lucitanib alone was 25.9 weeks (22.2 weeks in the $10 \mathrm{mg}$ dose level and 39.1 weeks in the $12.5 \mathrm{mg}$ dose level). The overall median lucitanib relative dose intensity (RDI) was $77.4 \%$ with two-thirds of patients having a RDI below $90 \%$. The median lucitanib RDI observed reflects the treatment interruptions and dose reductions proposed for the management of toxicities in the study protocol (Table 2).
Table 2 Treatment duration and compliance $(N=18)$
Table 3 Dose interruptions and dose reductions of lucitanib

\begin{tabular}{llll}
\hline & $10 \mathrm{mg}(N=12)$ & $12.5 \mathrm{mg}(N=6)$ & All $(N=18)$ \\
\hline $\begin{array}{l}\text { Global treatment duration (weeks) }{ }^{\mathrm{a}} \\
\quad\end{array}$ & 24.6 & & \\
$\quad$ Median & $8-68$ & 44.6 & 28.1 \\
Min-max & $7(58.3)$ & $6(100)$ & $8-83$ \\
$>24$ weeks $[n(\%)]$ & & & $13(72.2)$ \\
Lucitanib treatment duration (weeks) & 22.2 & 39.1 & 25.9 \\
$\quad$ Median & $3-66$ & $25-76$ & $3-76$ \\
Min-max & & & \\
Lucitanib relative dose intensity (\%) & 85.8 & 76.2 & 77.4 \\
$\quad$ Median & $54-100$ & $61-91$ & $54-100$ \\
Min-max & $7(58.3)$ & $5(83.3)$ & $12(66.7)$ \\
$\quad<90 \%[n(\%)]$ & $5(41.7)$ & $1(16.7)$ & $6(33.3)$ \\
{$[90-110 \%][n(\%)]$} & & & \\
\hline
\end{tabular}

$(\%): n /$ Nobs $\times 100$

$n$ number of patient in a class, $N$ number of patients by dose level

${ }^{\mathrm{a}}$ Global treatment duration took into account lucitanib (IMP) and fulvestrant (NIMP) intake duration

\begin{tabular}{|c|c|c|c|}
\hline & $10 \mathrm{mg}(N=12)$ & $12.5 \mathrm{mg}(N=6)$ & All $(N=18)$ \\
\hline \multicolumn{4}{|c|}{ Patients having (at least one) lucitanib interruption } \\
\hline$n(\%)$ & $12(100)$ & $6(100)$ & $18(100)$ \\
\hline \multicolumn{4}{|l|}{ Reason for interruption } \\
\hline Adverse event & $10(76.9)$ & $6(60.0)$ & 16 (69.6) \\
\hline Dosing error & - & $1(10.0)$ & $1(4.4)$ \\
\hline Other & $2(15.4)$ & $1(10.0)$ & $3(13.0)$ \\
\hline Progression of disease & - & $1(10.0)$ & $1(4.4)$ \\
\hline Subject non-compliance & $1(7.7)$ & $1(10.0)$ & $2(8.7)$ \\
\hline \multicolumn{4}{|c|}{ Patients having interruption $>14$ consecutive days } \\
\hline$n(\%)$ & $3(25.0)$ & $3(50.0)$ & $6(33.3)$ \\
\hline \multicolumn{4}{|c|}{ Reason of lucitanib interruption $>14$ consecutive days } \\
\hline Adverse event & $3(100)$ & $3(100)$ & $6(100)$ \\
\hline \multicolumn{4}{|c|}{ Patients having (at least one) lucitanib dose reduction } \\
\hline$n(\%)$ & $7(58.3)$ & $6(100)$ & $13(72.2)$ \\
\hline \multicolumn{4}{|l|}{ Reasons for dose reduction } \\
\hline Adverse event & $7(100)$ & $6(100)$ & $13(100)$ \\
\hline
\end{tabular}

$n$ number of patient in a category, $N$ number of patients by dose level 
All the patients required at least one lucitanib dose interruption, mostly due to adverse events (16/23 interruptions due to AEs, $69.6 \%$ ), while $13 / 18$ patients $(72.2 \%)$ had at least one dose reduction, all due to adverse events (Table 3 ).

\section{Safety}

A total of 365 emergent adverse events (EAEs) were observed and all 18 patients experienced at least one EAE. Overall, 216 out of the 365 total EAEs were considered to be related to lucitanib or/and fulvestrant (160 EAEs reported in all patients were considered as related to lucitanib only; 15 EAEs reported in $9 / 18$ patients [50.0\%] were considered as related to fulvestrant only; 41 EAEs in 7/18 patients [38.9\%] were considered as related to both lucitanib and fulvestrant). EAEs related to lucitanib only or both lucitanib and fulvestrant were mostly $(85.6 \%$ - 172/201 EAEs) graded $\leq 2$; $12.4 \%$ (25/201 EAEs) were graded 3 while three related EAEs $(1.5 \%-2$ cases of hypertensive crisis and 1 case of neutropenia) were graded 4, as worst grade. No fatal EAE related to IMP was reported. The most common related grade $\geq 3$ toxicities occurring in more than two patients were hypertension (78\%) and asthenia (22\%). EAEs related to lucitanib only or both lucitanib and fulvestrant and reported with an overall incidence of more than $20 \%$ are presented by worst grade in Table 4.

\section{Activity}

One patient $(8.3 \%)$ in the $10 \mathrm{mg} /$ day dose group and two (33.3\%) in the $12.5 \mathrm{mg} /$ day dose group achieved a partial response (PR) for an overall response rate of $16.7 \%$. All PRs were confirmed at a subsequent timepoint occurring at least 4 weeks after the first assessment of the response. For these 3 patients, the duration of response was 4.7, 13 and 14.8 months, respectively (Table 5).

\section{FGFR amplification and PD}

The IHC and FISH analyses were restricted to the patients with an available biopsy (archived tumor biopsies at diagnosis (optional for all patients) and/or metastatic biopsies (optional for patients of dose allocation)). In all, IHC and FISH data were available (i.e., non-missing) for $9 / 18$ patients and $8 / 18$ patients, respectively (Supplementary Table ${ }^{\circ} 1$ ).

The FGFRl gene was found to be amplified in one patient (ratio 7.7 and copy number 14.4). This patient who achieved SD as best overall response also displayed a high level of FGFR1 expression (global H-score of 150 and a membrane and cytoplasmic $\mathrm{H}$-score of 250 and 50, respectively) consistent with the amplification of FGFR1. Low FGFR1 expression (global $\mathrm{H}$-score $<50$ ) was observed for all other patients which were consistent with the absence of FGFRI amplification (evaluated by FISH).

Table 4 Treatment-related EAEs with an incidence more than $20 \%$ by worst grade

\begin{tabular}{|c|c|c|c|c|c|c|c|c|c|c|c|c|}
\hline \multirow[t]{2}{*}{ Primary SOC/PT } & \multicolumn{2}{|c|}{ Grade 1} & \multicolumn{2}{|c|}{ Grade 2} & \multicolumn{2}{|c|}{ Grade 3} & \multicolumn{2}{|c|}{ Grade 4} & \multicolumn{2}{|c|}{ Grade 5} & \multicolumn{2}{|c|}{ Any grade } \\
\hline & $n$ & $\%$ & $n$ & $\%$ & $n$ & $\%$ & $n$ & $\%$ & $n$ & $\%$ & $n$ & $\%$ \\
\hline \multicolumn{13}{|l|}{ Vascular disorders } \\
\hline Hypertension & - & - & 1 & 5.6 & 14 & 77.8 & - & - & - & - & 15 & 83.3 \\
\hline \multicolumn{13}{|l|}{ Endocrine disorders } \\
\hline Hypothyroidism & 1 & 5.6 & 13 & 72.2 & - & - & - & - & - & - & 14 & 77.8 \\
\hline \multicolumn{13}{|c|}{ Gastrointestinal disorders } \\
\hline Diarrhea & 4 & 22.2 & 2 & 11.1 & 1 & 5.6 & - & - & - & - & 7 & 38.9 \\
\hline Nausea & 4 & 22.2 & 2 & 11.1 & - & - & - & - & - & - & 6 & 33.3 \\
\hline Stomatitis & 4 & 22.2 & - & - & - & - & - & - & - & - & 4 & 22.2 \\
\hline \multicolumn{13}{|c|}{ General disorders and administration site conditions } \\
\hline Asthenia & 2 & 11.1 & 1 & 5.6 & 4 & 22.2 & - & - & - & - & 7 & 38.9 \\
\hline Fatigue & 2 & 11.1 & 2 & 11.1 & - & - & - & - & - & - & 4 & 22.2 \\
\hline \multicolumn{13}{|l|}{ Investigations } \\
\hline Weight decreased & 1 & 5.6 & 2 & 11.1 & 1 & 5.6 & - & - & - & - & 4 & 22.2 \\
\hline \multicolumn{13}{|c|}{ Metabolism and nutrition disorders } \\
\hline Decreased appetite & 4 & 22.2 & 1 & 5.6 & - & - & - & - & - & - & 5 & 27.8 \\
\hline \multicolumn{13}{|c|}{ Musculoskeletal and connective tissue disorders } \\
\hline Muscle spasms & 2 & 11.1 & 2 & 11.1 & - & - & - & - & - & - & 4 & 22.2 \\
\hline \multicolumn{13}{|c|}{ Renal and urinary disorders } \\
\hline Proteinuria & 2 & 11.1 & 3 & 16.7 & - & - & - & - & - & - & 5 & 27.8 \\
\hline
\end{tabular}


Table 5 Activity results

\begin{tabular}{|c|c|c|c|}
\hline & $10 \mathrm{mg}(N=12)$ & $12.5 \mathrm{mg}(N=6)$ & All $(N=18)$ \\
\hline \multicolumn{4}{|c|}{ Objective response rate ${ }^{a}$} \\
\hline$n(\%)$ & $1(8.3)$ & $2(33.3)$ & $3(16.7)$ \\
\hline $95 \% \mathrm{CI}^{\mathrm{c}}$ & {$[1.49 ; 35.39]$} & {$[9.68 ; 70.00]$} & {$[5.84 ; 39.22]$} \\
\hline \multicolumn{4}{|c|}{ Clinical benefit rate ${ }^{\mathrm{b}}$} \\
\hline$n(\%)$ & $4(33.3)$ & $6(100)$ & $10(55.6)$ \\
\hline $95 \% \mathrm{CI}^{\mathrm{c}}$ & {$[13.81 ; 60.94]$} & {$[60.97 ; 100.00]$} & {$[33.72 ; 75.44]$} \\
\hline \multicolumn{4}{|c|}{ Duration of clinical benefit (months) } \\
\hline Median & 6.5 & 16,5 & 9.2 \\
\hline $95 \% \mathrm{CI}$ & {$[6.4 ; 7.6]$} & {$[6.8 ; 18.4]$} & {$[6.4 ; 18.4]$} \\
\hline Min; $\max$ & $6.4 ; 7.6$ & $6.8 ; 18.4$ & $6.4 ; 18.4$ \\
\hline \multicolumn{4}{|c|}{ Time to first response (months) } \\
\hline Median & 1.89 & 3.59 & 3.56 \\
\hline Min; $\max$ & $1.89 ; 1.89$ & $3.56 ; 3.63$ & $1.89 ; 3.63$ \\
\hline \multicolumn{4}{|c|}{ Duration of response (months) } \\
\hline Median & 4.7 & 13.9 & 13 \\
\hline $95 \% \mathrm{CI}$ & {$[. ;]$.} & {$[13 ; 14.8]$} & {$[4.7 ; 14.8]$} \\
\hline Min; $\max$ & $4.7 ; 4.7$ & $13 ; 14.8$ & $4.7 ; 14.8$ \\
\hline \multicolumn{4}{|c|}{ Progression free survival (months) } \\
\hline Median & 5.5 & 16.6 & 6.5 \\
\hline $95 \%$ CI & {$[1.8 ; 6.5]$} & {$[6.8 ; 18.4]$} & {$[3.7 ; 7.6]$} \\
\hline Min; $\max$ & $1.7 ; 7.6$ & $6.8 ; 18.4$ & $1.7 ; 18.4$ \\
\hline
\end{tabular}

${ }^{a}$ Objective response rate (best overall response $=\mathrm{CR}$ or $\mathrm{PR}$ )

${ }^{\mathrm{b}}$ Clinical benefit rate (best overall response $=\mathrm{CR}$ or $\mathrm{PR}$ or stabilization $(\mathrm{SD}$ or non $\mathrm{CR} /$ non PD) $>24$ weeks or at the end of cycle 6)

${ }^{c}$ The Wilson method was used to calculate the $95 \%$ confidence interval of the estimate

Circulating protein analyses on changes from baseline were available in 16/18 patients (Supplementary Table $\mathrm{n}^{\circ} 2$ ). From baseline to C2D1, a statistically significant high increase was observed for PlGF $(+358 \%$ of change compared to median at baseline, adjusted $p$ value $=0.0003$ ). In addition, a statistically significant increase was observed for VEGF-A $(+47 \%$, adjusted $p$ values $=0.0114), \mathrm{IL}-8(+36 \%$, adjusted $p$ value $=0.0142)$ and FGF23 $(+30 \%$, adjusted $p$ value $=0.0047)$, while a trend towards an increase was observed for VEGF-D $(+11 \%$, adjusted $p$ value $=0.0439)$. Because there were too few patients in the dose level of $12.5 \mathrm{mg}(N=6)$ and in the group of responders $(N=3)$, no conclusion could be drawn regarding results by dose level or responders.

\section{Discussion}

The purpose of this phase Ib study was to evaluate the safety and tolerability of the combination of lucitanib with fulvestrant in postmenopausal patients with ER positive/HER-2 negative metastatic breast cancer, who have progressed during or after prior treatment with fulvestrant.
According to the design of the study, a dose expansion part (study part 2) was to follow the dose allocation part (study part 1). In the dose expansion part, 2 cohorts were to be opened: cohort A with FGF+ (receptor or ligand amplified) patients, and cohort B with $F G F$ non-amplified patients (14 patients were planned in each of these cohorts). However, this study was prematurely terminated with only three patients enrolled in part 2. Subsequent to this decision, the sponsor also decided to discontinue the development of lucitanib monotherapy in BC.

Based on a strong preclinical rationale, the expectation was that FGFR amplification would play the role of an oncogenic driver [3] and therefore selection on the basis of FGFR amplification could identify patients most likely to respond. However, recent clinical results do not fully support the oncogenic role of FGFR and patients with $F G F R$ amplification respond inconsistently to FGFR inhibitors [25]. In a phase I studies of dovitinib (TKI of FGFR1-3, VEGFR 1-3 and PDGFR) [26] and BGJ398 (a selective FGFR 1-3 TKI) [27], no objective response was observed in FGFR amplified breast cancer patients. Similarly, moderate activity was observed with BGJ398 [27] and dovitinib [28] in $F G F R$ amplified squamous non-small-cell lung cancer. These observations question the idea of $F G F R$ amplification 
as an oncogenic driver and a predictive factor of clinical benefit from treatment with FGFR inhibitors.

The role of FGF pathway as an oncogenic driver is further questioned by the results of a recently reported randomized, placebo-controlled phase II trial, that evaluated whether the dovitinib/fulvestrant combination would improve clinical outcome in ER positive, HER-2 negative advanced breast cancer that had progressed during or after prior endocrine treatment [29]. A total of 97 patients were enrolled including 32 presenting FGF pathway amplification (15 in the dovitinib arm and 17 in the placebo arm). This trial failed to demonstrate any benefit in favor of the combination vs. placebo. Median PFS was 5.5 months for both, which is quite similar to the PFS observed in the INES study ( 28 weeks for the overall population). The median PFS was 10.9 months and 5.5 months in the $F G F$ pathway-amplified subgroup and 5.5 months and 5.5 months in the $F G F$ pathway-non-amplified subgroup for the dovitinib and placebo arms, respectively. However, this difference should be interpreted with caution given the small number of patients. The interpretation of the predictive role of $F G F$ pathway amplification is further complicated by the fact that overall response rate with dovitinib was higher in the $F G F$ pathway-non-amplified subgroup (ORRs for dovitinib vs. placebo were $20.0 \%$ vs. $12.5 \%$ in the $F G F$ pathway-amplified subgroup and $31.3 \%$ vs. $8.8 \%$ in the $F G F$ pathway-non-amplified subgroup). An exploratory analysis yielded no correlation between the $F G F R 1$ copy number and response to dovitinib in the $F G F$ pathway-amplified subgroup, although patient population analyzed was small.

Above mentioned clinical data [29] plus preclinical experiments completed by the sponsor (Unpublished Data) failed to confirm the hypothesis that addition of lucitanib would reverse sensitivity to endocrine treatments. Moreover, data from other lucitanib monotherapy studies in breast cancer demonstrated moderate activity compared to standard of care and failed to confirm a predictive role for $F G F R$ amplification [EudraCT nos. 2013-000288-10 (FINESSE) and NCT02109016 (US Breast)]. On that basis, a decision was taken to prematurely terminate the study.

Overall, the majority of treatment-related EAEs were mild to moderate with the exception of hypertension and asthenia that were severe (grade $\geq 3$ ) in $78 \%$ and $22 \%$ of patients, respectively. The AEs reported are known reported AEs of lucitanib, related to its anti-angiogenic mode of action [22]. No new toxicities were observed in this study. Out of nine patients enrolled in the $10 \mathrm{mg} /$ day dose level, only one patient developed a DLT (grade 3 hypertension) while none of the six patients enrolled in the $12.5 \mathrm{mg} /$ day dose level developed a DLT. Based on the MCRM, a dose level of $15 \mathrm{mg} /$ day should have been tested next. However, data coming from a pooled analysis of five lucitanib studies ongoing at the time (EudraCT nos. 2013-000288-10
(FINESSE), 2010-019121-34 (FIH), 2013-001520-19 (INES) and 2013-003874-29 (Lung) and NCT02109016 (US Breast)], suggested that the $10 \mathrm{mg} /$ day would be a better tolerated dose (EAEs appeared to be less frequent at $10 \mathrm{mg}$, especially grade 3 hypertension). This observation was further supported by the fact that all patients required a dose interruption, mainly due to adverse events. Moreover, $72 \%$ $(13 / 18)$ of the total patient population and $100 \%(6 / 6)$ of patients in the $12.5 \mathrm{mg} /$ day dose level required at least one dose reduction. All these observations highlight the fact that treatment with the combination requires close patient monitoring and intensive management of adverse events. On the basis of these safety considerations, we decided to stop the dose allocation (part 1 of the study) and to use $10 \mathrm{mg} /$ day of lucitanib as the recommended dose (given in combination with fulvestrant) for part 2 of the study.

Despite the early termination of this study, evidence of efficacy was observed in this cohort of heavily pretreated (two-thirds of patients have received $\geq 3$ previous regimens for metastatic disease) patients. Indeed ORR was $16.7 \%$ and clinical benefit rate (defined as CR/PR/SD for $\geq 24$ weeks) was $55.6 \%$. Moreover, median duration of response was 13 months, while two of the three responding patients had a response that lasted more than 1 year. Finally, median PFS was 6.5 months (and 16.6 months in the $12.5 \mathrm{mg}$ cohort). The PFS observed in this study is comparable to what is observed with palbociclib in a similar setting [30], although results observed in this study should be interpreted with caution given the premature termination and the small number of patients included.

A potential explanation is that the clinical activity observed with lucitanib and dovitinib was due to their antiangiogenic mode of action. Indeed, in the first in human study of lucitanib [22], clinical activity was observed in an angiogenesis-sensitive cohort of patients (defined as patients that responded or had stable disease for at least 6 months to an anti-angiogenic-based treatment, or a tumor type known to be potentially sensitive to anti-angiogenic therapy). Complete responses were observed in patients with thyroid cancer, a tumor type that is considered to be sensitive to anti-angiogenic treatment. Moreover, the observed toxicity profile is consistent with the expected toxicity profile of a potent anti-angiogenic agent, with hypertension, asthenia and proteinuria frequently observed, adverse events that are typical of the blockade of the VEGF axis. Lenvatinib, a FGFR1-4, VEGFR1-3, KIT, RET and PDGF alpha inhibitor with pharmacological properties similar to lucitanib has reported positive phase III data in thyroid and hepatocellular cancer, two anti-angiogenesis-sensitive tumor types [31,32].

In cell assays, lucitanib is highly potent on VEGFR2, FGFR1-3, PDGFR- $\alpha$ and $\beta$. In this study, it has been observed that lucitanib was able to significantly increase FGF23 serum levels, indicative of FGFR-1 inhibition [26, 
33]. Because there was too few patients in the group of responders $(N=3)$, no conclusion could be drawn regarding FGF23 serum levels as a marker of response. Interestingly for dovitinib, the level of FGF23 increase was similar in all groups regardless of FGFR1 or HR status, suggesting that FGF23 is a surrogate pharmacodynamic marker of FGFR1 signaling inhibition [29]. Statistically significant increases of VEGF-A as well as others angiogenesis biomarkers (PIGF and VEGF-D) were consistent with results observed in other lucitanib studies and with the anti-angiogenic mode of action of the compound.

Further investigation is required to better understand the role of FGF pathway as a potential oncogenic driver, and the potential role of FGFR inhibition in reversing resistance to endocrine treatment to establish the settings where this class of drugs can offer a meaningful clinical benefit.

Acknowledgements We would like to thank all the patients that participated in the study and the teams involved in all sites and at Servier. We also would like to thank the team that performed the pharmacodynamic experiments at Institut de Recherches Servier (Catherine de Montrion, Carole Chotard, Johann Richard, Amélie Pontisso-Mahout, Agnès Chomel).

Funding This study was funded by I.R.I.S (Institut de Recherches Internationales Servier) (ISRCTN23201971).

\section{Compliance with ethical standards}

Conflict of interest M. Campone has received consulting fees from Novartis, Lilly, Servier, Abbvie, AstraZeneca and Sanofi. T. Bachelot has received consulting fees and research grants from AstraZeneca, Novartis and Roche and honorarium from Novartis and Roche. F. Penault-Llorca has received consulting fees from Roche, AstraZeneca, MSD, BMS, Novartis, Pfizer, Sanofi and research grants from Roche and AstraZeneca. F. Andre has received research grants from AstraZeneca, Lilly, Novartis and Pfizer and travel/accommodations reimbursements from GlaxoSmithKline, Novartis and Roche. A. Pallis, V. Agrapart, M. J. Pierrat, C. Poirot, F. Dubois and L. Xuereb are employee of Institut de Recherches Internationales Servier. C. J. Bossard, N. Guigal-Stephan and B. Lockhart are employee of Institut de Recherches Servier.

OpenAccess This article is distributed under the terms of the Creative Commons Attribution 4.0 International License (http://creativeco mmons.org/licenses/by/4.0/), which permits unrestricted use, distribution, and reproduction in any medium, provided you give appropriate credit to the original author(s) and the source, provide a link to the Creative Commons license, and indicate if changes were made.

\section{References}

1. Brady N, Chuntova P, Bade LK, Schwertfeger KL (2013) The FGF/FGFR axis as a therapeutic target in breast cancer. Expert Rev Endocrinol Metab 8:391-402

2. Haugsten EM, Wiedlocha A, Olsnes S, Wesche J (2010) Roles of fibroblast growth factor receptors in carcinogenesis. Mol Cancer Res 8:1439-1452
3. Turner N, Grose R (2010) Fibroblast growth factor signalling: from development to cancer. Nat Rev Cancer 10:116-129

4. Andre F, Job B, Dessen P, Tordai A, Michiels S, Liedtke C, Richon C, Yan K, Wang B, Vassal G, Delaloge S, Hortobagyi GN, Symmans WF, Lazar V, Pusztai L (2009) Molecular characterization of breast cancer with high-resolution oligonucleotide comparative genomic hybridization array. Clin Cancer Res $15: 441-451$

5. Kadota M, Sato M, Duncan B, Ooshima A, Yang HH, Diaz-Meyer N, Gere S, Kageyama S, Fukuoka J, Nagata T, Tsukada K, Dunn BK, Wakefield LM, Lee MP (2009) Identification of novel gene amplifications in breast cancer and coexistence of gene amplification with an activating mutation of PIK3CA. Cancer Res 69:7357-7365

6. Moelans CB, de Weger RA, Monsuur HN, Vijzelaar R, van Diest PJ (2010) Molecular profiling of invasive breast cancer by multiplex ligation-dependent probe amplification-based copy number analysis of tumor suppressor and oncogenes. Mod Pathol 23:1029-1039

7. Adnane J, Gaudray P, Dionne CA, Crumley G, Jaye M, Schlessinger J, Jeanteur P, Birnbaum D, Theillet C (1991) BEK and FLG, two receptors to members of the FGF family, are amplified in subsets of human breast cancers. Oncogene 6:659-663

8. Jacquemier J, Adelaide J, Parc P, Penault-Llorca F, Planche J, deLapeyriere O, Birnbaum D (1994) Expression of the FGFR1 gene in human breast-carcinoma cells. Int J Cancer 59:373-378

9. Courjal F, Cuny M, Simony-Lafontaine J, Louason G, Speiser P, Zeillinger R, Rodriguez C, Theillet C (1997) Mapping of DNA amplifications at 15 chromosomal localizations in 1875 breast tumors: definition of phenotypic groups. Cancer Res 57:4360-4367

10. Letessier A, Sircoulomb F, Ginestier C, Cervera N, Monville F, Gelsi-Boyer V, Esterni B, Geneix J, Finetti P, Zemmour C, Viens P, Charafe-Jauffret E, Jacquemier J, Birnbaum D, Chaffanet M (2006) Frequency, prognostic impact, and subtype association of $8 \mathrm{p} 12,8 \mathrm{q} 24,11 \mathrm{q} 13,12 \mathrm{p} 13,17 \mathrm{q} 12$, and $20 \mathrm{q} 13$ amplifications in breast cancers. BMC Cancer 6:245

11. Karlsson E, Waltersson MA, Bostner J, Perez-Tenorio G, Olsson B, Hallbeck AL, Stal O (2011) High-resolution genomic analysis of the 11q13 amplicon in breast cancers identifies synergy with 8p12 amplification, involving the mTOR targets S6K2 and 4EBP1. Genes Chromosomes Cancer 50:775-787

12. Gelsi-Boyer V, Orsetti B, Cervera N, Finetti P, Sircoulomb F, Rouge C, Lasorsa L, Letessier A, Ginestier C, Monville F, Esteyries S, Adelaide J, Esterni B, Henry C, Ethier SP, Bibeau F, Mozziconacci MJ, Charafe-Jauffret E, Jacquemier J, Bertucci F, Birnbaum D, Theillet C, Chaffanet M (2005) Comprehensive profiling of 8p11-12 amplification in breast cancer. Mol Cancer Res 3:655-667

13. Elbauomy ES, Green AR, Lambros MB, Turner NC, Grainge MJ, Powe D, Ellis IO, Reis-Filho JS (2007) FGFR1 amplification in breast carcinomas: a chromogenic in situ hybridisation analysis. Breast Cancer Res 9:R23

14. Turner N, Lambros MB, Horlings HM, Pearson A, Sharpe R, Natrajan R, Geyer FC, van KM, Kreike, Mackay B, Ashworth A, van de Vijver A, Reis-Filho MJ JS (2010) Integrative molecular profiling of triple negative breast cancers identifies amplicon drivers and potential therapeutic targets. Oncogene 29:2013-2023

15. Wilkerson PM, Reis-Filho JS (2013) The 11q13-q14 amplicon: clinicopathological correlations and potential drivers. Genes Chromosomes Cancer 52:333-355

16. Shi M, Linnartz R, Versace R, Graus Porta D, Kay A, Dugan M (2011) Dovitinib (TKI258), a dual inhibitor of FGFR and VEGFR, induces tumor growth suppression in xenograft models of primary human breast cancer. Cancer Res 71(Suppl 24):P3-17-11 
17. Shiang CY, Qi Y, Wang B, Lazar V, Wang J, Fraser SW, Hortobagyi GN, Andre F, Pusztai L (2010) Amplification of fibroblast growth factor receptor-1 in breast cancer and the effects of brivanib alaninate. Breast Cancer Res Treat 123:747-755

18. Turner N, Pearson A, Sharpe R, Lambros M, Geyer F, LopezGarcia MA, Natrajan R, Marchio C, Iorns E, Mackay A, Gillett C, Grigoriadis A, Tutt A, Reis-Filho JS, Ashworth A (2010) FGFR1 amplification drives endocrine therapy resistance and is a therapeutic target in breast cancer. Cancer Res 70:2085-2094

19. Balko JM, Mayer IA, Sanders ME, Miller TW, Kuba MG, Meszoely IM, Wagle N, Garraway LA, Arteaga CL (2012) Discordant cellular response to presurgical letrozole in bilateral synchronous ER+ breast cancers with a KRAS mutation or FGFR1 gene amplification. Mol Cancer Ther 11:2301-2305

20. Formisano L, Stauffer KM, Young CD, Bhola NE, GuerreroZotano AL, Jansen VM, Estrada MM, Hutchinson KE, Giltnane JM, Schwarz LJ, Lu Y, Balko JM, Deas O, Cairo S, Judde JG, Mayer IA, Sanders M, Dugger TC, Bianco R, Stricker T, Arteaga CL (2017) Association of FGFR1 with ERalpha maintains ligand-independent ER transcription and mediates resistance to estrogen deprivation in ER+ breast cancer. Clin Cancer Res 23(20):6138-6150

21. Lucitanib; Clinical Investigator's Brochure. Version $n^{\circ}$ 05: $16 \mathrm{AD}$

22. Soria JC, DeBraud F, Bahleda R, Adamo B, Andre F, Dienstmann R, Delmonte A, Cereda R, Isaacson J, Litten J, Allen A, Dubois F, Saba C, Robert R, D'Incalci M, Zucchetti M, Camboni MG, Tabernero J (2014) Phase I/IIa study evaluating the safety, efficacy, pharmacokinetics, and pharmacodynamics of lucitanib in advanced solid tumors. Ann Oncol 25:2244-2251

23. ICH Efficacy Guidelines.E6(R1) (2009) Good clinical practice: consolidated guideline: 2017

24. Eisenhauer EA, Therasse P, Bogaerts J, Schwartz LH, Sargent D, Ford R, Dancey J, Arbuck S, Gwyther S, Mooney M, Rubinstein L, Shankar L, Dodd L, Kaplan R, Lacombe D, Verweij J (2009) New response evaluation criteria in solid tumors: revised RECIST guideline (version 1.1). Eur J Cancer 45:228-247

25. Pearson A, Smyth E, Babina IS, Herrera-Abreu MT, Tarazona N, Peckitt C, Kilgour E, Smith NR, Geh C, Rooney C, Cutts R, Campbell J, Ning J, Fenwick K, Swain A, Brown G, Chua S, Thomas A, Johnston SRD, Ajaz M, Sumpter K, Gillbanks A, Watkins D, Chau I, Popat S, Cunningham D, Turner NC (2016) Highlevel clonal FGFR amplification and response to FGFR inhibition in a translational clinical trial. Cancer Discov 6:838-851

26. Andre F, Bachelot T, Campone M, Dalenc F, Perez-Garcia JM, Hurvitz SA, Turner N, Rugo H, Smith JW, Deudon S, Shi M, Zhang Y, Kay A, Porta DG, Yovine A, Baselga J (2013) Targeting FGFR with dovitinib (TKI258): preclinical and clinical data in breast cancer. Clin Cancer Res 19:3693-3702
27. Nogova L, Sequist LV, Perez Garcia JM, Andre F, Delord JP, Hidalgo M, Schellens JH, Cassier PA, Camidge DR, Schuler M, Vaishampayan U, Burris H, Tian GG, Campone M, Wainberg ZA, Lim WT, LoRusso P, Shapiro GI, Parker K, Chen X, Choudhury S, Ringeisen F, Graus-Porta D, Porter D, Isaacs R, Buettner R, Wolf J (2017) Evaluation of BGJ398, a fibroblast growth factor receptor 1-3 kinase inhibitor, in patients with advanced solid tumors harboring genetic alterations in fibroblast growth factor receptors: results of a global phase I, dose-escalation and doseexpansion study. J Clin Oncol 35:157-165

28. Paik PK, Shen R, Berger MF, Ferry D, Soria JC, Mathewson A, Rooney C, Smith NR, Cullberg M, Kilgour E, Landers D, Frewer P, Brooks N, Andre F (2017) A phase Ib open-label multicenter study of AZD4547 in patients with advanced squamous cell lung cancers. Clin Cancer Res 23(18):5366-5373

29. Musolino A, Campone M, Neven P, Denduluri N, Barrios CH, Cortes J, Blackwell K, Soliman H, Kahan Z, Bonnefoi H, Squires M, Zhang Y, Deudon S, Shi MM, Andre F (2017) Phase II, randomized, placebo-controlled study of dovitinib in combination with fulvestrant in postmenopausal patients with $\mathrm{HR}+$. Breast Cancer Res 19:18

30. Turner NC, Ro J, Andre F, Loi S, Verma S, Iwata H, Harbeck N, Loibl S, Huang BC, Zhang K, Giorgetti C, Randolph S, Koehler M, Cristofanilli M (2015) Palbociclib in hormone-receptor-positive advanced breast cancer. N Engl J Med 373:209-219

31. Schlumberger M, Tahara M, Wirth LJ, Robinson B, Brose MS, Elisei R, Habra MA, Newbold K, Shah MH, Hoff AO, Gianoukakis AG, Kiyota N, Taylor MH, Kim SB, Krzyzanowska MK, Dutcus CE, de Las HB, Zhu J, Sherman SI (2015) Lenvatinib versus placebo in radioiodine-refractory thyroid cancer. N Engl J Med 372:621-630

32. Cheng AL, Finn RS, Qin S, Han KH, Ikeda K, Piscaglia F, Baron AD, Park JW, Han G, Jassem J, Blanc JF, Vogel A, Komov D, Evans TRJ, Lopez C, Ductus C, Ren M, Krajevic S, Tamai T, Kudo M (2017) Phase III trial of lenvatinib (LEN) vs sorafenib (SOR) in first-line treatment of patients (pts) with unresectable hepatocellular carcinoma (uHCC). J Clin Oncol 35(suppl 15):4001

33. Gattineni J, Bates C, Twombley K, Dwarakanath V, Robinson ML, Goetz R, Mohammadi M, Baum M (2009) FGF23 decreases renal $\mathrm{NaPi}-2 \mathrm{a}$ and $\mathrm{NaPi}-2 \mathrm{c}$ expression and induces hypophosphatemia in vivo predominantly via FGF receptor 1 . Am J Physiol Ren Physiol 297:F282-F291

Publisher's Note Springer Nature remains neutral with regard to jurisdictional claims in published maps and institutional affiliations. 